\title{
Nectin-4 and DNA mismatch repair proteins expression in upper urinary tract urothelial carcinoma (UTUC) as a model for tumor targeting approaches: an ImGO pilot study
}

Maria Letizia Calandrella ${ }^{*}$, Simona Francesconi², Cecilia Caprera², Claudia Mosillo ${ }^{2}$, Claudia Caserta', Diana Giannarelli ${ }^{3}$, Matteo Corsi ${ }^{2}$, Serena Macrini ${ }^{1}$, Annalisa Guida ${ }^{1}$, Stefano Ascani ${ }^{2}$ and Sergio Bracarda ${ }^{1}$

\begin{abstract}
Background: Upper urinary tract urothelial carcinoma (UTUC) accounts for only about 5-10\% of all urothelial cancers and is characterized by an aggressive and frequently rapidly fatal behavior. However, detailed knowledge of its molecular profile is still lacking.

Materials and methods: We identified, by chart analysis, patients who underwent radical nephroureterectomy or diagnostic biopsy for UTUC between January 2015 and August 2020 at the Santa Maria Hospital of Terni, in Italy. Eligible patients were required to have also adequate clinical informations and follow-up details. The primary objective of the study was to evaluate DNA mismatch repair (MMR) proteins and Nectin-4 immunohistochemical expression in UTUC, looking also for an eventual correlation between these molecular features. The secondary objective was to investigate genomic instability in the case of a MMR protein loss. Expression of proteins was assessed by using immunohistochemistry and microsatellite instability (MSI) performed by next generation sequencing. Nectin-4 expression was reported using an intensity scoring system (score, $0-3+$ ), instead the expression of DNA MMR proteins was indicated as present (no loss) or not present (loss).
\end{abstract}

Results: Thirty four cases have been evaluated and 27 considered eligible for the study with their tumor samples analyzed. Nectin-4 was found to be expressed in $44 \%$ of cases and $18.5 \%$ of patients showed defective-MMR phenotype. We found a significant correlation between Nectin-4 expression and MSH2/MSH6 protein loss. Out of 7 patients with DNA MMR proteins loss or equivocal phenotype, 3 showed MSI.

Conclusions: Our pilot study suggest a possible relationship between Nectin-4 and DNA MMR protein expression in UTUC and a clinically significant correlation between defective MMR phenotype and genomic instability. Because of the possible implications of these data for innovative treatment approaches, the need for further studies in this area is warranted.

Keywords: Upper tract urothelial carcinoma, Nectin-4, DNA mismatch repair proteins, MSH2/MSH6 loss, Microsatellite instability

*Correspondence: marialetiziacalandrella@gmail.com

${ }^{1}$ Medical and Translational Oncology Unit, Department of Oncology,

Azienda Ospedaliera Santa Maria, Terni, Italy

Full list of author information is available at the end of the article

\section{Background}

Upper urinary tract urothelial carcinoma (UTUC) is a rare and heterogeneous disease, representing about $5-10 \%$ of all urothelial tumors with limited evidence in 
literature but some emerging biologic details $[1,2]$. From the clinical point of view, two-thirds of UTUC patients have an invasive disease at diagnosis and $7 \%$ of them present metastatic disease [3]. UTUC has an aggressive behavior, with a five-year survival rate of only $60 \%$ compared to $77 \%$ for all stage urothelial cancer patients [4]. Although similar histological characteristics have been found between UTUC and lower tract carcinoma (LTUC, mainly bladder cancer), increasing evidence suggest UTUC as a distinct biologic disease with specific genetic and epigenetic features [5].

UTUC represents the third most common tumor (5\%) in the group of hereditary nonpolyposis colorectal cancer syndrome (HNPCC) related tumors, after colon (63\%) and endometrial cancer (9\%) [6]. HNPCC, known as Lynch Syndrome, is an autosomal dominant genetic condition associated with germ-line mutations affecting one or several MMR genes (MSH2, MLH1, MSH6, PMS2, EPCAM) with consequent DNA mismatch repair (MMR) enzyme activity lost and increased genomic instability (microsatellite instability - MSI). Of note, while MSI is rare in bladder cancer (3\% of cases), a defective DNA MMR system has been documented in more than $15 \%$ of sporadic UTUC cases, to reiterate their different genetic profiles [7].

Nectin- 4 is a type I transmembrane protein involved in $\mathrm{Ca} 2+-$ indipendent cellular adhesion. It is considered a strongly promising biomarker and a specific therapeutic target for urothelial tumors. A moderate to strong staining for Nectin-4 was observed in more than $60 \%$ of urothelial cancer and $53 \%$ of breast tumor cases, with a consequent convincing rationale for drug development in these tumors $[8,9]$. Recent data from phase II and III trials showed a significant efficacy of Enfortumab Vedotin (EV), an antibody-drug conjugate targeting Nectin-4, in patients with urothelial cancers previously treated with platinum and anti-programmed death 1 or anti-programmed death ligand 1 (PD-1/PD-L1) therapies $[10,11]$.

Aim of this pilot study is to describe the molecular profile of UTUC and investigate eventual correlation between immunohistochemical (IHC) expression of Nectin-4 and DNA mismatch repair (MMR) proteins (MLH1, MHS2, MSH6 and PMS2) in UTUC samples. Moreover, we performed an MSI analysis in patients with MMRdeficient (dMMR) or equivocal phenotype.

\section{Materials and methods}

\section{Population and tissue samples}

We identified, by chart analysis, patients who underwent to a radical nephroureterectomy or diagnostic biopsy for UTUC between January 2015 and August 2020 in our Hospital. Eligible patients were required to have histological samples from their primary tumors or metastatic sites, adequate clinical information, and follow-up details. Appropriate approval was obtained from the local institutional review board and Ethical Committee (CER-Umbria - Protocol Number: 005594, 29 Sept 2020). Written informed consent was obtained from all alive patients.

\section{IHC analysis of Nectin- 4 and MMR proteins expression}

Tumor samples were obtained from formalin-fixed and paraffin-embedded surgical specimens. After deparaffinization, rehydration, and the blocking of endogenous peroxidase activity, immunohistochemical staining was performed. For MMR protein expression we used a monoclonal mouse anti-MSH2 antibody (clone G219-1129), a monoclonal rabbit anti-MSH6 antibody (clone SP93), a monoclonal mouse anti-MLH1 antibody (clone M1), and a monoclonal mouse anti-PSM2 antibody (clone A16-4), all using the Ventana Benchmark autostaining system. MMR protein "loss" is defined by the absence of IHC staining in the nucleus of tumor cells. Any nuclear staining was considered as "no loss" of expression.

Regarding Nectin-4 expression we used a rabbit polyclonal antibody (clone ab155692). The intensity of Nectin-4 expression was determined using the histochemical scoring system (H-score) which is defined as the sum of product of the staining intensity $(0 ; 1 ; 2 ; 3)$ and the percentage of stained cells at each intensity level (0-100). The final score ranges from 0 to 300 . Finally, the samples were classified as negative (0; $\mathrm{H}$-score, $0-14)$, low (1+; H-score, 15-99), medium (2+; H-score, 100-199), and high (3+; H-score, 200-300). Microscopic slides from all the specimens were reviewed by a urologic pathologist.

\section{Molecular analysis of microsatellite instability}

Serial 8- $\mu \mathrm{m}$ histological sections of formalin-fixed, paraffin-embedded tissue blocks of normal $(\mathrm{N})$ and tumor $(\mathrm{T})$ were prepared using DNA histology precautions. Normal or tumor tissue was microdissected from unstained slides for each case by overlaying the unstained slide onto the H\&E stained side. Dissection of unstained slides was performed in a laminar flow hood after UV irradiation. Only samples with a tumour cell content of at least $25 \%$, which corresponded to the limit of detection determined for the multiplex PCR assay, were included. MSI assays were performed on microdissected DNA, extracted using the "MagCore Genomic DNA FFPE One-Step" Kit (Diatech Pharmacogenetics). Microvolume samples were quantified by a spectrophotometer system, "NanoDrop One Microvolume UV-Vis Spectrophotometer (Thermo Fisher Scientific). Paired DNA from normal and tumor tissue specimens were amplified by multiplex amplification PCR (Titano MSI Kit, Diatech Pharmacogenetics) with fluorescent primers (Bethesda panel: BAT25, BAT26, 
D2S123, D17S250, D5S346 and BAT40, D18S58, NR21, NR24, TGFßRII) and subsequent DNA fragment analysis on an automated sequencer. Instability is determined by fragment size analysis on a capillary electrophoresis instrument (SeqStudio Genetic Analyzer, Thermo Fisher Scientific) following PCR amplification of DNA from a patient's normal and tumor tissue samples. The microsatellite status of each sample was determined based on percentage of unstable loci. The status was defined as MSI-high (MSI-H) when 4 or more markers displayed instability and MSI-low (MSI-L) when 1 to 3 markers exhibited instability. A sample was classified as microsatellite stable (MSS) when no MSI was found.

\section{Statistical analysis}

Patient characteristics were reported using descriptive statistics such as mean and standard deviation for quantitative variables and absolute and percentages frequencies for qualitative variables. The association between observed data was evaluated with Fisher exact test. $P$-values $<0.05$ were considered statistically significant. The analyses were performed using the statistical software IBM-SPSS v. 21.0.

\section{Results}

\section{Descriptive analysis}

A total of 34 patients were evaluated and 27 were included in the final analysis (3 not available clinical information and 4 not adequate histological samples); the clinicopathological characteristics of patients are summarized in Table 1. Median age of patients at diagnosis was 70 years-old, with a male/female ratio of $8: 1$. Pyelocaliceal tumors were approximately twice as common as ureteral tumors (ratio 1.5:1). About pathological stage, we have identified mostly pT3 (40.7\%) and pNx (92.6\%). The $14.8 \%$ of patients $(4 / 27)$ underwent platinum-based adjuvant chemotherapy and $18.5 \%$ (5/27) had family history of cancer and second primitive cancers.

\section{Analysis of IHC features}

The overall results are summarized in the Table 2.

\section{Nectin-4}

The patterns of Nectin-4 IHC expression are shown in Fig. 1.

Nectin- 4 was detected in $44.4 \%$ of cases $(12 / 27)$ with a variable expression spectrum (High 0\%; Medium 17.8\%; Low 25.9\%; Negative 51.9\%; NV 3.7\%).

\section{MMR deficiency}

The patterns of MMR proteins IHC expression are shown in Fig. 2.
Table 1 Clinicopathological characteristics of patients

\begin{tabular}{|c|c|}
\hline & $\mathrm{N}(\%)$ \\
\hline \multicolumn{2}{|l|}{ GENDER } \\
\hline M & $24(88.9)$ \\
\hline $\mathrm{F}$ & $3(11.1)$ \\
\hline AGE (median - range) & $70(44-86)$ \\
\hline METASTATIC AT DIAGNOSIS & $4(14.8)$ \\
\hline CONCURRENT BLADDER CANCER & $8(29.6)$ \\
\hline METACHRONOUS TUMOURS IN LOWER URINARY TRACT & $19(70.4)$ \\
\hline \multicolumn{2}{|l|}{ LOCATION } \\
\hline Pelvis & $16(59.3)$ \\
\hline Urether & $11(40.7)$ \\
\hline SURGERY & $26(96.3)$ \\
\hline \multicolumn{2}{|l|}{ PATHOLOGICAL T STAGE } \\
\hline Ta & $1(3.7)$ \\
\hline $\mathrm{T} 1$ & $10(37.0)$ \\
\hline $\mathrm{T} 2$ & $5(18.5)$ \\
\hline $\mathrm{T} 3$ & $11(40.7)$ \\
\hline \multicolumn{2}{|l|}{ PATHOLOGICAL N STAGE } \\
\hline No & $1(3.7)$ \\
\hline N1 & $1(3.7)$ \\
\hline NX & $25(92.6)$ \\
\hline \multicolumn{2}{|l|}{ GRADE } \\
\hline High & $19(70.4)$ \\
\hline Low & $8(29.6)$ \\
\hline \multicolumn{2}{|l|}{ LYMPHOVASCULAR INVASION } \\
\hline Yes & $4(14.8)$ \\
\hline No & $23(85.2)$ \\
\hline \multicolumn{2}{|l|}{ HISTOLOGY } \\
\hline Urothelial & $24(88.9)$ \\
\hline Squamous cell carcinoma & $3(11.1)$ \\
\hline SMOKING & $22(81.5)$ \\
\hline PRESENCE OF A SECOND PRIMARY CANCER & $5(18.5)$ \\
\hline FAMILY HISTORY OF CANCER & $5(18.5)$ \\
\hline PROFESSIONAL RISK FACTOR EXPOSURE & $12(44.4)$ \\
\hline ADJUVANT CHEMOTHERAPY & $4(14.8)$ \\
\hline
\end{tabular}

The $18.5 \%$ of patients $(5 / 27)$ showed dMMR phenotype characterized in all cases by loss of MSH2 and MSH6 proteins. Two patients had a "dual lose" profile (MSH2/ MSH6 loss): one patient has a loss of expression of 3/4 proteins (MSH2/MSH6/PMS2), and two patients the loss of expression of all 4 proteins (MSH2/MSH6/MLH1/ PMS2). In two cases IHC MSH2 expression analysis gave an equivocal result.

Cases with expression of Nectin-4 and DNA MMR protein loss The $5 \mathrm{dMMR}$ cases expressed Nectin-4 with profile of expression Low-Medium and had a pyelocaliceal localization. The median age of this group was 67 years-old 
Table 2 Immunohistochemical features

\begin{tabular}{ll}
\hline & N (\%) \\
\hline NECTIN4 & \\
NE & $1(3.7)$ \\
Negative & $14(51.9)$ \\
Low & $7(25.9)$ \\
Medium & $5(17.8)$ \\
High & 0 \\
MLH1 & \\
Equivocal & $1(3.7)$ \\
Negative & $2(7.4)$ \\
Positive & $24(88.9)$ \\
MSH2 & \\
Equivocal & $2(7.4)$ \\
Negative & $5(18.5)$ \\
Positive & $20(74.1)$ \\
MSH6 & \\
Equivocal & $1(3.7)$ \\
Negative & $6(22.2)$ \\
Positive & $20(74.1)$ \\
PMS2 & \\
Equivocal & $1(3.7)$ \\
Negative & $3(11.1)$ \\
Positive & $23(85.2)$ \\
\hline Nor & $5(18.5)$ \\
\hline
\end{tabular}

${ }^{a}$ Not evaluable

(range 57-82); $2 / 5$ had a personal history of second cancers (breast and colon) and 3/5 had a family cancer history (Table 3).

\section{Descriptive analysis of microsatellite}

The analysis of microsatellite on DNA extracted from tumor tissue related to cases of $\mathrm{dMMR}$ or equivocal phenotype, showed MSI in 3/7 cases: one case MSI-H (8/10 loci) and two cases MSI-L (1/10 e 3/10 loci respectively). The remaining cases analyzed were MSS.

\section{Statistical correlations}

The results of the statistical analysis showed a joint frequency between expression of Nectin-4 and loss of MSH2/MSH6 with statistical significance $(p=0.014$ and $p=0.015$ respectively). However, Nectin-4 expression was not significantly associated with MLH1 $(p=0.48)$ and PMS2 $(p=0.22)$. The $60 \%$ of patients with MSH2/ MSH6 protein loss presented a positive family history for cancer with statistical correlation $(p=0.05$ and $p=0.09$ respectively). The loss of the protein $\mathrm{MSH} 2$ has been found associated also to female sex $(p=0.09)$.

\section{Discussion}

Our pilot study reports a clinical and molecular profile of UTUC outlining its own biological identity in respect to LTUC. Regarding clinical features, our data confirmed that about $40 \%$ of UTUC patients presented with a locally advanced cancer (pT3) at diagnosis, of note almost all cases had not received a lymphadenectomy (LND) at the time of surgery (pNx 96\%), confirming the low trend of this surgical procedure in daily clinical practice, even in the case of bulky primary tumors. Consistently, Moschini et al. evaluated a series of 1512 UTUC patients treated with RNU and reported that $64 \%$ of cases did not received a concomitant LND, many of them undergoing laparoscopic surgery. In the rare cases in which LND was performed, the procedure was frequently inadequate in terms of number of lymph nodes

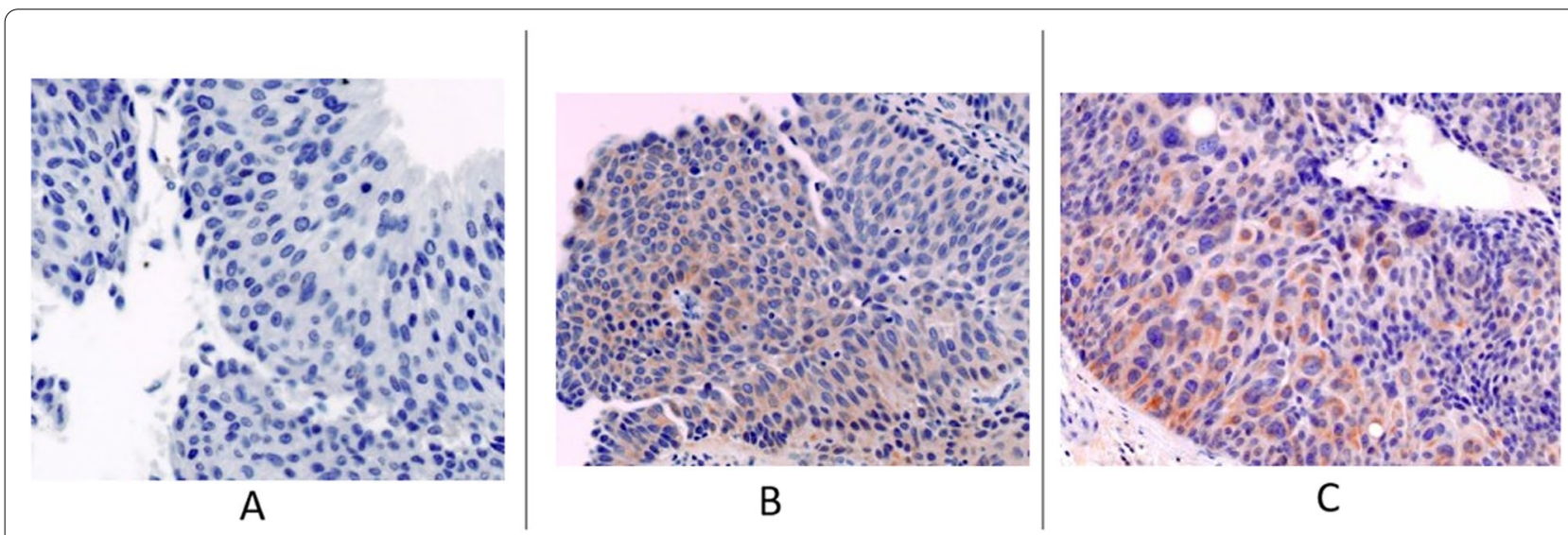

Fig. 1 Immunohistochemical expression of Nectin4 in UTUC specimens (A) Tissue with negative expression. B Tissue with low expression. C Tissue with medium expression 

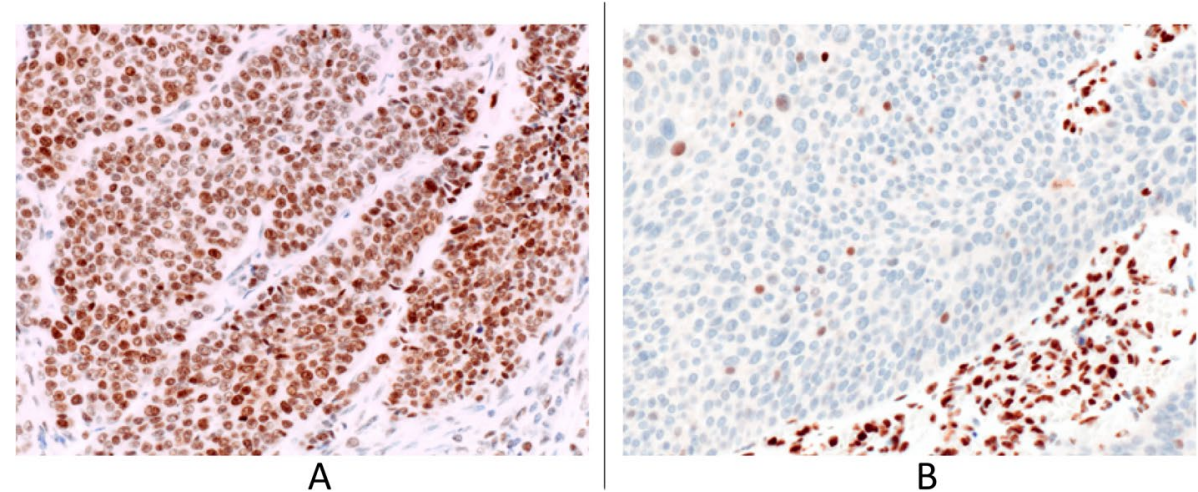

Fig. 2 Immunohistochemical expression of MSH6 in UTUC specimens. A MSH6 positive with MSS. B MSH negative with MSI

Table 3 Clinicopathological characteristics of patients with dMMR profile

\begin{tabular}{|c|c|c|c|c|c|c|c|}
\hline CASE & 1 & 2 & 3 & 4 & 5 & 6 & 7 \\
\hline $\begin{array}{l}\text { SECOND PRIMITIVE } \\
\text { CANCER }\end{array}$ & COLON & / & BREAST & / & / & / & / \\
\hline FAMILY HISTORY & $\begin{array}{l}\text { DAUGHTER (RECTAL } \\
-37 \text { y.o.) MOTHER } \\
\text { (RENAL) }\end{array}$ & $\begin{array}{l}\text { FATHER (LUNG) } \\
\text { MOTHER(OVARIAN) } \\
\text { BROTHER (PANCRE- } \\
\text { ATIC } 47 \text { y.o.) }\end{array}$ & / & / & $\begin{array}{l}\text { MATHER(ENDOMETRIAL) } \\
\text { MATERNAL GRANDFA- } \\
\text { THER (COLON) } \\
\text { MATERNAL AUNT (ENDO- } \\
\text { METRIAL) } \\
\text { MATERNAL COUSIN } \\
\text { (ENDOMETRIAL) }\end{array}$ & / & / \\
\hline HISTOLOGY & $U C^{a}$ & $U C$ & UC & UC & UC & $U C$ & $U C$ \\
\hline AGE & 65 & 62 & 70 & 82 & 57 & 55 & 64 \\
\hline SITE & PELVIS & PELVIS & PELVIS & PELVIS & URETHER & URETHER & URETHER \\
\hline MSS/MSI & MSS & MSS & MSS & MSS & MSI-H & MSI-L & MSI-L \\
\hline NECTIN-4 & MEDIUM & LOW & MEDIUM & LOW & LOW & MEDIUM & NEGATIVE \\
\hline MMR lost & MSH2/MSH6 & MSH2/MSH6/PMS2 & $\begin{array}{l}\text { MLH1/ } \\
\text { MSH2/ } \\
\text { MSH6/PMS2 }\end{array}$ & $\begin{array}{l}\text { MLH1/ } \\
\text { MSH2/ } \\
\text { MSH6/PMS2 }\end{array}$ & MSH2/MSH6 & EQUIVOCAL & EQUIVOCAL \\
\hline
\end{tabular}

${ }^{a}$ Urothelial carcinoma

removed with consequences for optimal staging and cancer specific survival $[12,13]$. Moreover, an accurate staging may allow the indication to adjuvant chemotherapy and a more appropriate schedule of follow-up [14].

The expression of Nectin-4, a potential innovative predictive factor, was identified in $44 \%$ of cases, mainly represented by low-medium expression levels. The phase II trial EV-201 with Enfortumab vedotin enrolled patients with locally advanced urothelial carcinoma previously treated with PD-1/PD-L1 inhibitor and/or platinumcontaining chemotherapy. An overall positive expression of Nectin-4 was shown in $97 \%$ of the cases (UTUC and LTUC). Therefore, out of 125 patients, 35\% had a UTUC but disjoint IHC data are not available [10]. The analysis of Nectin-4 expression (2394 cases of cancers from 34 different histology), published by Challita-Eid and colleagues, showed that $69 \%$ of cancer specimens were positive for Nectin-4. In particular, it has been identified in $83 \%$ of bladder cancer tissues (High 31\% - Moderate $29 \%$ - Low 23\% - Negative 17\%) [8]. However, this evaluation was carried out exclusively on bladder carcinomas. Contrarily, Tomiyama et al. showed a Nectin-4 expression in $65 / 99(65.7 \%)$ of UTUC samples with a predominant low-medium expression profile $(1+, 31.3 \% ; 2+$, $24.2 \%$; and $3+, 10.1 \%$ ) [15], confirming, as in our data, a lower expression of Nectin-4 In UTUC in respect to LTUC, and a partially different biological profile.

In the present pilot study, we identified a d-MMR phenotype in $18.5 \%$ of the cases. Previous studies have shown a frequency between 4.6 and $27.5 \%$ in UTUC populations not otherwise selected $[16,17]$. This wide range may depend on the IHC method, the number of samples, and the geographical origin. Moreover, all dMMR cases described were characterized by MSH2/MSH6 
proteins loss, outcome consistent with published analysis (70-100\%) [18]. Of interest, in the limits of our relatively small sample size, we observed a statistically significant correlation between Nectin-4 expression and MSH2/ MSH6 loss ( $p=0.009$ e $p=0.005$ respectively), indeed all patients with dMMR phenotype showed a Nectin-4 expression.

Pembrolizumab was the first drug to be approved with a tumor-agnostic indication for patients with unresectable or metastatic, MSI-H or dMMR solid tumors [19, 20]. However, in the published trials cases with UTUC were under-represented (and only $2.1 \%$ of the cases were urothelial carcinomas). In the phase III study Keynote 045 , evaluating pembrolizumab versus second line chemotherapy in recurrent advanced urothelial cancer, UTUC cases represent $14 \%$ of the entire population [21]. This study showed a superior survival benefit of pembrolizumab versus the standard of care in all subgroup. Likewise, a case of high-grade UTUC treated with immunotherapy was reported, the patient experienced a partial response (90\% shrinkage) after five courses of Pembrolizumab [22]. These experiences suggest a way forward for the use of immune check-point inhibitors in UTUC, but also highlight the importance of a proper patient selection.

Our IHC data about MMR protein expression allowed us to carry out a pre-screening and estimate the prevalence of cases potentially associated with a Lynch syndrome. The median age of the identified 7 patients was 65 years old, compared to 70 years of the study entire population. The median age of onset Lynch-related colon cancer is 45 years-old, about 20-30years younger in respect to the sporadic forms and it is shown that in patients with Lynch syndrome, UTUC occurs 15 years after other associated oncologic diseases [23, 24]. By using the clinical criteria to screen the risk of a hereditary disease, only $3 / 7$ of patients with dMMR met the classical clinical criteria. Accordingly, many hereditary forms of UTUC may be misclassified as sporadic and their incidence under-estimated with a consequent lack of genetic counseling [25]. Incidence of MSI is about 3\% in urothelial bladder but higher in UTUC, up to $15 \%$ of the cases [26, 27]. We investigated dMMR phenotype as a surrogate for MSI with a matching rate of $42 \%$. One of the 7 examined resulted MSI-H (8/10 loci) and two cases resulted MSI-L (1/10 and 3/10 loci respectively), and the remaining 4 a MSS phenotype. Data about an eventual correlation rate between the two methods for UTUC are lacking in the literature [28]. However, recent studies showed a rate of concordance of 97.8 and $96.9 \%$ for colon and endometrial cancers, respectively [29, 30]. Therefore, more studies with larger samples are needed to assess a possible interchangeability for these data.
The present analysis has several limitations and should be considered only as a preliminary pilot study testing some possible specific details of UTUC cases. First, its retrospective nature, second, the low number of included cases, even considering that UTUC is a rare subpopulation of urothelial cancer. Third limitation point is that data concerning hereditary syndromes should be confirmed by DNA sequencing. Finally, ICH evaluations are operator-dependent and have some analytical variability.

This preliminary experience suggests a way forward for the clinical and molecular characterization of this rare subtype of urothelial cancer to improve knowledge on potential predictive factors and design innovative treatment approaches, also in respect to LTUC.

\section{Conclusion}

Nectin-4 and MMR could be expressed at different levels in UTUC in respect to urothelial bladder cancer. The observed pattern of combination of these data may be tested as a potential predictive tool for the use of a combination of immune- drug conjugates, such as Enfortumab vedotin, and checkpoint inhibitors. The need for further studies in this area is warranted.

\section{Abbreviations \\ UTUC: Upper tract urothelial carcinoma; MMR: DNA mismatch repair; MSI: Microsatellite instability; LTUC: lower tract urothelial carcinoma; HNPCC: Hereditary nonpolyposis colorectal cancer; EV: ENfortumab-Vedotin; PD1/ PDL1: Programmed death 1 or anti-programmed death ligand 1; IHC: Immu- nohistochemistry; dMMR: Deficient DNA Mismatch Repair; MSI-H: Microsatel- lite Instability High; MSI-L: Microsatellite Instability Low; MSS: Microsatellite Stability; LND: Lymphadenectomy.}

\section{Supplementary Information}

The online version contains supplementary material available at https://doi. org/10.1186/s12885-022-09259-z.

Additional file 1.

\section{Acknowledgements}

Not Applicable.

\section{Authors' contributions}

All authors have read and approved the final manuscript. Conceptualization, S.B.. and ML.C..; methodology, S.F., C.C1., M.C..; formal analysis, D.G..; data curation, ML.C., S.M, C.M, A.G...; writing-original draft preparation, ML.C., S.B..; writing-review and editing, ML.C, S.B., C.M., C.C2, S.A. All Authors read and approved the manuscript.All authors agreed to the published version of the manuscript.

\section{Funding}

This research received no external funding.

\section{Availability of data and materials}

The datasets used and/or analyzed during the current study are available from the corresponding author on reasonable request. 


\section{Declarations}

\section{Ethics approval and consent to participate}

All methods were carried out in accordance with relevant guidelines and regulations. Appropriate approval was obtained from the local institutional review board and Ethical Committee (CER-Umbria - Protocol Number: 005594, 29 Sept 2020). Written informed consent was obtained from all alive patients. Informed consent was obtained from legal guardian/LAR's of participants which were not alive/dead during sampling.

\section{Consent for publication}

Not Applicable.

\section{Competing interests}

The authors declare no conflict of interest.

\section{Author details}

${ }^{1}$ Medical and Translational Oncology Unit, Department of Oncology, Azienda Ospedaliera Santa Maria, Terni, Italy. ${ }^{2}$ Pathology Unit, Department of Medicine, Medical Clinic Section and Anatomical Pathology, Azienda Ospedaliera Santa Maria, Terni, Italy. ${ }^{3}$ Biostatistical Unit, Regina Elena National Cancer Institute, IRCCS, Rome, Italy.

Received: 4 October 2021 Accepted: 27 January 2022

Published online: 14 February 2022

\section{References}

1. AIOM-AIRTUM I numeri del cancro in Italia 2020.

2. Rouprêt M, Babjuk M, Burger $M$, et al. European Association of Urology guidelines on upper urinary tract urothelial carcinoma: 2020 update. Eur Urol. 2021:79(1):62-79.

3. Mbeutcha A, Rouprêt M, Kamat AM, et al. Prognostic factors and predictive tools for upper tract urothelial carcinoma: a systematic review. World J Urol. 2017;35(3):337-53.

4. Rouprêt M, Hupertan V, Seisen T, et al. Prediction of cancer specific survival after radical nephroureterectomy for upper tract urothelial carcinoma: development of an optimized postoperative nomogram using decision curve analysis. J Urol. 2013;189(5):1662-9.

5. Green DA, Rink M, Xylinas E, et al. Urothelial carcinoma of the bladder and the upper tract: disparate twins. J Urol. 2013;189(4):1214-21.

6. Rouprêt $M$, Yates DR, Comperat E, Cussenot O. Upper urinary tract urothelial cell carcinomas and other urological malignancies involved in the hereditary nonpolyposis colorectal cancer (lynch syndrome) tumor spectrum. Eur Urol. 2008;54(6):1226-36.

7. Rouprêt M, Azzouzi AR, Cussenot O. Microsatellite instability and transitional cell carcinoma of the upper urinary tract. BJU Int. 2005;96(4):489-92.

8. Challita-Eid PM, Satpayev D, Yang P, et al. Enfortumab Vedotin antibodydrug conjugate targeting Nectin-4 is a highly potent therapeutic agent in multiple preclinical cancer models. Cancer Res. 2016;76(10):3003-13.

9. McGregor BA, Sonpavde G. Enfortumab Vedotin, a fully human monoclonal antibody against Nectin 4 conjugated to monomethyl auristatin E for metastatic urothelial carcinoma. Expert Opin Investig Drugs. 2019;28(10):821-6.

10. Rosenberg JE, O'Donnell PH, Balar AV, et al. Pivotal trial of Enfortumab Vedotin in urothelial carcinoma after platinum and anti-programmed death 1/programmed death ligand 1 therapy. J Clin Oncol. 2019;37(29):2592-600.

11. Powles T, Rosenberg JE, Sonpavde GP, et al. Enfortumab Vedotin in previously treated advanced urothelial carcinoma. N Engl J Med. 2021;384(12):1125-35.

12. Moschini M, Foerster B, Abufaraj M, et al. Trends of lymphadenectomy in upper tract urothelial carcinoma (UTUC) patients treated with radical nephroureterectomy. World J Urol. 2017;35(10):1541-7.

13. Dominguez-Escrig JL, Peyronnet B, Seisen T, et al. Potential benefit of lymph node dissection during radical Nephroureterectomy for upper tract urothelial carcinoma: a systematic review by the European
Association of Urology guidelines panel on non-muscle-invasive bladder cancer. Eur Urol Focus. 2019;5(2):224-41.

14. Birtle A, Johnson M, Chester J, et al. Adjuvant chemotherapy in upper tract urothelial carcinoma (the POUT trial): a phase 3, open-label, randomised controlled trial. Lancet. 2020;395(10232):1268-77.

15. Tomiyama E, Fujita K, Rodriguez Pena MDC, et al. Expression of Nectin-4 and PD-L1 in upper tract Urothelial carcinoma. Int J Mol Sci. 2020;21(15):5390 Published 2020 Jul 29.

16. Ericson $\mathrm{KM}$, Isinger $\mathrm{AP}$, Isfoss $\mathrm{BL}$, Nilbert MC. Low frequency of defective mismatch repair in a population-based series of upper urothelial carcinoma. BMC Cancer. 2005;5:23 Published 2005 Mar 1.

17. García-Tello A, Ramón de Fata F, Andrés G, Ropero S, López Jl, Angulo JC. DNA repair genes and prognosis in sporadic forms of urothelial carcinoma of the upper urinary tract. Actas Urol Esp. 2014;38(9):600-7.

18. Ito T, Kono K, Eguchi H, et al. Prevalence of Lynch syndrome among patients with upper urinary tract carcinoma in a Japanese hospital-based population. Jpn J Clin Oncol. 2020;50(1):80-8.

19. Le DT, Kim TW, Van Cutsem E, et al. Phase II open-label study of Pembrolizumab in treatment-refractory, microsatellite instability-high/mismatch repair-deficient metastatic colorectal cancer: KEYNOTE-164. J Clin Oncol. 2020;38(1):11-9.

20. Marabelle A, Le DT, Ascierto PA, et al. Efficacy of Pembrolizumab in patients with noncolorectal high microsatellite instability/mismatch repair-deficient cancer: results from the phase II KEYNOTE-158 study. J Clin Oncol. 2020:38(1):1-10.

21. Bellmunt J, de Wit R, Vaughn DJ, et al. Pembrolizumab as secondline therapy for advanced urothelial carcinoma. N Engl J Med. 2017;376(11):1015-26.

22. Ikarashi D, Kitano S, Ishida K, et al. Complete pathological response to Neoadjuvant Pembrolizumab in a patient with Chemoresistant upper urinary tract Urothelial carcinoma: a case report. Front Oncol. 2020;10:564714 Published 2020 Sep 24.

23. Umar A, Boland CR, Terdiman JP, et al. Revised Bethesda guidelines for hereditary nonpolyposis colorectal cancer (Lynch syndrome) and microsatellite instability. J Natl Cancer Inst. 2004:96(4):261-8.

24. Crockett DG, Wagner DG, Holmäng S, Johansson SL, Lynch HT. Upper urinary tract carcinoma in Lynch syndrome cases. J Urol. 2011:185(5):1627-30.

25. Audenet F, Colin P, Yates DR, et al. A proportion of hereditary upper urinary tract urothelial carcinomas are misclassified as sporadic according to a multi-institutional database analysis: proposal of patient-specific risk identification tool. BJU Int. 2012:110(11 Pt B):E583-9.

26. Amira N, Rivet J, Soliman H, et al. Microsatellite instability in urothelial carcinoma of the upper urinary tract. J Urol. 2003;170(4 Pt 1):1151-4.

27. Hartmann A, Zanardo L, Bocker-Edmonston T, et al. Frequent microsatellite instability in sporadic tumors of the upper urinary tract. Cancer Res. 2002;62(23):6796-802.

28. Harper HL, McKenney JK, Heald B, et al. Upper tract urothelial carcinomas: frequency of association with mismatch repair protein loss and lynch syndrome. Mod Pathol. 2017;30(1):146-56

29. Bartley AN, Luthra R, Saraiya DS, Urbauer DL, Broaddus RR. Identification of cancer patients with Lynch syndrome: clinically significant discordances and problems in tissue-based mismatch repair testing. Cancer Prev Res (Phila). 2012;5(2):320-7.

30. Pérez-Carbonell L, Ruiz-Ponte C, Guarinos C, et al. Comparison between universal molecular screening for Lynch syndrome and revised Bethesda guidelines in a large population-based cohort of patients with colorectal cancer. Gut. 2012;61(6):865-72.

\section{Publisher's Note}

Springer Nature remains neutral with regard to jurisdictional claims in published maps and institutional affiliations. 